\title{
Modular Change in Platform Ecosystems and Routine Mirroring in Organizations
}

\author{
Stefan Seidel \\ University of Liechtenstein \\ stefan.seidel@uni.li
}

\author{
Thomas Grisold \\ University of Liechtenstein \\ thomas.grisold@uni.li
}

\author{
Nicholas Berente \\ University of Notre Dame \\ nberente@nd.edu
}

\begin{abstract}
Organizational routines involve modular digital technologies that are part of larger platform ecosystems that often transcend organizational boundaries. Change in organizational routines is thus interwoven with innovation and associated change in digital platforms. To get at this "embedded" routine change, we use the concept of modular operators to conceptualize how changes to digital technologies in platform ecosystems are mirrored in changes in the organizational routines in which these technologies are implicated. We distinguish between enabling and constraining impacts and develop a set of propositions to move towards a theory of "routine mirroring." We use the Industrial Internet of Things (IIoT) as a base example.
\end{abstract}

Keywords: platform ecosystems, organizational routines, organizational change, modularity, modular operators

\section{Introduction}

Information technologies are enacted in organizational routines $[1,2]$. Because of their modularity and recombinability, information technologies can be enacted in different routines in different ways across context and time- - leading some to conclude that we live in the "Lego era" [3]. This development is fueled by the emergence of platform ecosystems - networks of innovation that produce complements which create network effects and make digital platforms more valuable [4]. Digital platforms are systems that provide essential functionality as a foundation for the development of complementary products, technologies, and services [5]. In the past, organizations were largely in control of the modular structure of the software system they were using, since it was typically locally hosted. With the advent of platform ecosystems, this is no longer the case, because routines increasingly involve digital technologies that are part of larger platform ecosystems. Examples of such platform ecosystems include operating systems (e.g., iOS, Windows, Android), applications in the form of web browsers (e.g., Firefox, Edge) or ERP systems (e.g., SAP, Microsoft Dynamics), web platforms for various purposes (e.g., Facebook), or Internet of Things (IoT) solutions (e.g., Thingworx, Microsoft Azure, or the Salsforce IoT Cloud). In contemporary organizing, change in organizational routines is thus interwoven with the platform ecosystems that the organization takes part in - and hence with change in that platform ecosystem. Organizational routines are embedded in broader ecosystems and theory is required that explains how ecosystem change and routine change are interrelated.

The Industrial Internet of Things (IIoT) provides our base example. IIoT configurations involve smart devices [6] with digital capabilities that are embedded in broader ecosystems. These ecosystems evolve - elements are added, updated, or removed from these ecosystems. Examples include: a new module for predictive maintenance hosted in a cloud; a new production machine with sensors and actuators; a new sensor that is added to a machine and provides a new data stream; a new bridge that can connect hitherto unconnected devices to the networked system. Such components can be enacted in organizational routines and be involved in changing performances of these routines. These examples also highlight that the modular choices an organization can make can have both physical and digital elements. New machines with sensors and actuators need to be physically installed, but a module for predictive maintenance can relatively simply be enacted in organizational routines by connecting sensors to the cloud.

Changes to routines can be continuous and occasionally even disruptive, and can pertain to the platform's core functionality as well as to the modules that are available through the platform. Participating in only one platform ecosystem (i.e. connecting all devices through one platform) might even come at 
the risk of complete network failure if the platform is discontinued.

Further, we can distinguish between enabling and constraining ecosystem impacts on routines. On the one hand, changes in the ecosystem can provide novel affordances that can be enacted in organizational routines [7] — such as in cases where new modules are added to the ecosystem. On the other hand, changes in the ecosystem can create constraints on organizational routines - such as in cases where components are eliminated or changed in the ecosystem.

Our goal in this paper is to theorize about the relationship between platform ecosystems and organizational routines. Both platform ecosystems and organizations are complex systems, and to theorize about their interrelationships we turn to the concept of modular operators. They allow us to attend to specific modular changes at the platform level and how these are related to modular changes at the routine level. Our work thus bridges recent theorizing on platform ecosystems to the literature on organizational routines, in particular, and organizing in general.

We proceed as follows. The next section introduces the research background in terms of organizational routines and artifacts in organizational routines, the emergent relevance of platform ecosystems, and modular operators. Our conceptual framework development and development of propositions along with some illustrations ensue. We conclude by discussing our findings in light of existent literature.

\section{Research Background}

\subsection{Routines and Artifacts}

Organizational routines are repetitive and recognizable patterns of actions that are carried out by multiple actors [9]. Routines are at the heart of organizing [10]. They provide an organization with stability and at the same time they are also a source of change. Routines consist of ostensive aspects that provide models of and for a routine for different actors, and a performative aspect that refers to the specific actions taken by specific actors at specific points in time [2]. The ostensive aspect guides the performative aspect, and the performances influence the ostensive aspect. This recursive relationship creates a generative dynamic whereby routines evolve over time [11].

Central to routines theory is that actors have agency and that routines are mindfully accomplished [12]. In principle, actors are free to choose how they act and behave, for example, by not following rules.
In practice, however, actors typically follow rules and enact routines by-and-large in a way that is consistent with the goals and interests of the organization [13]. In light of this tension, researchers have been interested in the role of artifacts in routines. Artifacts are seen as mediators between the ostensive and the performative aspects of routines [9]. To some extent, artifacts inscribe a logic by design - they incorporate rules and assumptions about how organizational work should be carried out [1].

In modern organizations, digital technologies are important artifacts, and are embedded within routines of all sorts [7]. Often, digital artifacts are not passive intermediaries but they are "obligatory points of passage" [1] that participate in knowledge co-creation and in the performance of actions [1]. Digital artifacts can introduce material aspects that can transform actors' roles, mindsets, and worldviews, and lead to "technology-mediated organizational change" [14]. For example, it has been observed that the introduction of an ERP systems changed a routine in different ways; some actions could not be enacted anymore because they were no longer supported by the system while other actions emerged due to new functionalities [14]. Similarly, features of Microsoft Excel sheets can change tasks that are associated with specific roles and coordination patterns among actors [15].

At the same time, since artifacts are "subverted and transformed through ongoing routine performance" [16], it cannot be determined how actors will use them [3]. Further, actors sometimes creatively navigate multiple organizational goals and enact routines in ways that reconcile competing imperatives [17]. Therefore, artifacts can indeed provide action affordances and enable and constrain specific routine performances $[15,16,18,19]$, but the impact of artifacts on a routine is not deterministic and human actors have multiple degrees of freedom to enact artifacts (or not) in their routines [12].

Although much of the literature on routines describes organizational systems and productivity technologies that operate within organizational boundaries [20], increasingly digital artifacts are implicated in broader platform ecosystems.

\subsection{Platform Ecosystems}

A platform is "the extensible codebase of a software-based system that provides core functionality shared by the modules that interoperate with it and the interfaces through which they interoperate" [21]. That is, key components are (a) the platform, (b) its interfaces, (c) the modules, and (d) the environment 
in which the platform operates, including competing ecosystems [21].

Software platforms use modular architectures that allow 'outsiders' - i.e., secondary developers [22] to make contributions, enabled by platform interfaces [23] in terms of software development kits (SDKs) and application programmers' interfaces (APIs). This, in turn, leads to the emergence of platform ecosystems as networks of innovation. Facebook, for instance, offers different SDKs and APIs for various applications, including machine learning, gaming, and augmented reality. That is, platforms are, from their very beginning, designed for ongoing augmentation-a key property of a system to allow for initially small systems built by a relatively small team to grow [8]. Platform owners exert control to ensure the interoperability of components such as apps-for instance, they screen what extensions they allow into their ecosystem [23]. Balancing control of the platform owner and autonomy of developers are key research issues [21].

Conceptual key to platform ecosystems is their layered modular architecture where products can be both platforms and products - the iPad, for instance, is a product, but also a platform enabling other firms to add modules and increase its value [24]. Layered architectures that, for instance, separate service and content layers allow third parties to add to the platform [24].

Thus, layered modular architectures are a central characteristic of platform ecosystems [11]. This architecture involves layered, recombinable components that extend beyond traditional organizational boundaries and go beyond traditional organizational systems and productivity tools. Exploring the implications of modularity for routine enactment is important in two respects. First, modularity is an effortful process that is constantly challenged and negotiated-it is deeply entangled with social and material practices [22], and thus, changes to the modular structures can lead to intended and unintended changes in the enactment of routines. Second, understanding modularity is interesting to explore routine dynamics because modules establish configurations among actors and tasks that lead to stable patterns of interactions, and thereby, they might provide "valuable insights into routine micro-dynamics" [1].

Thus one can expect changes in modular components of digital technologies to be reflected in the routine. After all, modularity is becoming a central feature of routines because they increasingly rely on systems that are useless in isolation but, when brought together, can constitute new organizational forms [3]. But, how, specifically, are different forms of modular change enacted in changes to routines?
Existing research does not offer an answer to this question. Thus, the specific goal of this paper is to build theory on the relationship between modular changes to digital technologies in platform ecosystems and the organizational routines in which they are implicated. To do so, we draw on Baldwin and Clark's [7] modular operators to think through how different sorts of modular change will be reflected in changes to organizational routines.

\subsection{Modular Operators}

The modular layered architecture of platform ecosystems allows comparably easy changes, compared with technological change of monolithic software systems with deep vertical integration. Changes typically involve the addition and removal of components, and can involve fairly minor incremental changes. But the aggregate of small changes can have a dramatic impact on the platform overall. Also, occasional changes to the platform's core functionality and its interfaces, such as with product updates, can lead to changes of a variety of types across a range of magnitudes.

To get at the changes in these ecosystems, and how they relate to changes in organizational routines, we turn to the concept of modular operators suggested by Baldwin and Clark in their seminal book on design rules [8]. Modular operators help explain the "dynamic possibilities that are inherent in modular structures"- they describe "'things that designers do' to a modular system" [8]. Baldwin and Clark identify six such operators:

1. splitting one module into more than one module

2. substituting one module design for another

3. augmenting - adding a new module to the system

4. excluding a module from the system

5. inverting modular interfaces

6. porting a module to another system

These six operators can be applied to analyze (a) the evolutionary trajectory of platform ecosystems and (b) how this evolution allows for change in routines through their enactment at the level of the organization. This is consistent with the application of modular operators by both architects and users of systems [8]. We can thus distinguish between changes in the platform ecosystem (e.g., modules are added to the platform and the platform ecosystem is thus augmented) and changes in organizational systems that draw on that platform ecosystem as they enact modules from that ecosystem in various ways. 


\section{Framework Development: Modular Change \& Routine Mirroring}

We theorize about how changes in a platform ecosystem (described in terms of the application of modular operators), translate into change in organization (also described in terms of the application of modular operators) as modules are enacted in organizational routines.

We describe the mechanism by which changes in platform ecosystems lead to changes in organizational routines as a process of routine mirroring. Routine mirroring describes the process by which modular changes in a platform ecosystem are enacted in organizational routines.

On this view, organizational routines mirror the platform ecosystem/the various platform ecosystems the organization participates in. In our base example of the Industrial Internet of Things, for instance, various organizations may use instances of the same module for predictive maintenance hosted in a cloud-if these organizations enact this component, they mirror this ecosystem component at the level of organizational routines.

In some cases, the availability of modules requires physical implementation on the organization's hardware devices (e.g., local installations of a machine with sensors and actuators), in other cases the availability requires a cloud solution. Logically, however, in both cases ecosystem modules become part of the organization's modular software architecture. In order for organizational change to occur, these modules must be enacted through activities performed by organizational actors, leading to routine change.

We use the notion of affordance and constraint [7] to get at this relationship between modular changes at the ecosystem level and associated changes at the level of organizational routines. Affordances describe the action possibilities that digital technologies provide to groups of users and that are capitalized on as organizational actors enact these action possibilities under consideration of action goals. Technology affordances are relations between the material features of technology and user groups [25] — broadly, they are relationships between modules and user groups. Affordances help explain how software modules are enacted within organizational routines [7]. This perspective of affordances as action potentials allows us to recognize that changes in the ecosystem are only mirrored if newly arising affordances are identified and enacted. Constraints, on the other hand, describe how the achievements of an action goal is restricted by available technology [7], or even the absence of technology (e.g., in cases where modules are removed from the ecosystem).

Figure 1 visualizes the general idea of how modular changes in platform ecosystems translate into modular changes at the level of organizational routines as the organization adopts and enacts certain modules. For illustrative purposes, Figure 1 shows two such change trajectories: First the exclusion of a module from the ecosystem that is mirrored at the level of the organization through exclusion from a routine because the module was enacted in Routine 1. Second, the augmentation of the ecosystem by adding a module, which in this case is mirrored as the module is enacted within both Routine 1 and Routine 2.

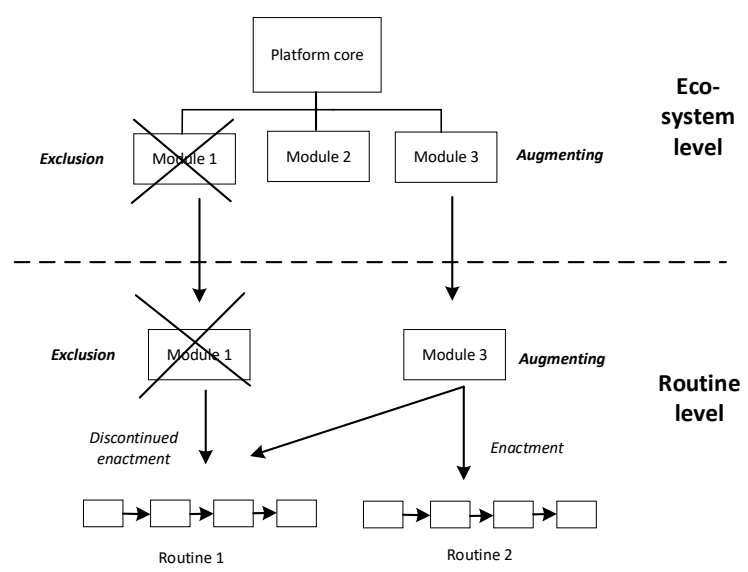

Figure 1. Modular Change and Routine Mirroring

The example illustrates how we can distinguish between changes in the platform ecosystem that afford new action potentials and changes that create constraints for organizational routines. Adding a module to the ecosystem might, for instance, lead to the identification of new affordances and, in turn, to routine change if those affordances are continuously enacted. On this view, the change in the ecosystem provides opportunities for routine change. On the other hand, excluding modules from the ecosystem can involve the discontinued availability of affordances and thus requiring to change a routine or the technology used within that routine in order to maintain the ability to achieve the routine's goals. We distinguish two categories of ecosystemembedded change - enabling and constraining. We use these two categories to present our propositions in what follows. 


\subsection{Enabling Ecosystem Impact}

Opportunities for routine change originating in the platform ecosystem occur if new affordances arise as new or changed ecosystem modules are interpreted in light of organizational action goals. Organizational change occurs if the organization adopts the respective modules and enacts them through organizational routines (i.e., mirrors the ecosystem change). This, in turn, leads to change the ostensive aspects of organizational routines if the change in performances is continuous. The technology-enacting routine becomes reified.

First, if the ecosystem's capabilities are augment$e d$ by adding a module, this creates the opportunity to adopt the new module and enact it within organizational routines. The decision to adopt the new module can have different reasons, including automatic software updates or the explicit decision to adopt the module because new affordances are identified. This adoption of the new module leads to organizational change when the module's affordances are enacted [7]. In this case, the organization interprets new features in light of a given institutional context and associated action goals [26]. This is perhaps the most typical type of ecosystem-embedded change, as ecosystems are based upon the idea that modules are added to the ecosystem to increase the ecosystem's value. This has given rise to a host of complementary innovations that have been adopted by organizations. Correspondingly:

Proposition 1a: Augmenting platform ecosystems (adding a new module to the system) leads to routine change if the new module provides affordances that are enacted in one or more organizational routines, thereby augmenting those routines.

An example for enabling change through augmenting the platform ecosystem is when the availability of new computing power in a cloud such as the module for predictive maintenance is adopted by the organization and enacted in the organization's monitoring routines as part of the IIoT system.

Second, if ecosystem modules are split-i.e., a design with interdependent parameters is converted into a hierarchical design with separate, independent modules [8] - these new modules might be adopted by the organization. Examples for such change can be found when software solution providers decide to split monolithic software systems, or more generally complex modules, into separate modules and associated services. Correspondingly:
Proposition 1b: Splitting of ecosystem modules leads to routine change if the split leads to the availability of new modules that provide affordances that are enacted in one more organizational routines.

For instance, if a new component to visualize production data that used to be part of a costly monolithic system is made available as a module, it may be be adopted by the organization to improve a specific monitoring routine.

Substitute modules may be made available in the ecosystem that provide additional affordances or improved versions of existing affordances. On this view, substitution is a natural complement of splitting, as splitting provides the ground for substituting modules of finer granularity [8]. Correspondingly:

Proposition 1c: Providing substitute ecosystem modules (i.e., alternative modules for organizations to choose from) leads to routine change if the substitution provides new affordances that are enacted in one or more organizational routines.

The availability of such substitutes, and thus the possibility for organizations to substitute modules, is typical for open platform ecosystems where there is competition within the ecosystem [23]. For instance, there may be different competing modules for predictive maintenance available. The same competition can also exist among different competing platform ecosystems the organization participates in or might opt to participate in.

Similarly, inversion can lead to the availability of new modules that may be adopted by an organization. Generally, inversion is the process by which a design is separated from its original context, and is made available for further use [8]. Hence:

Proposition 1d: Making new modules visible through inverting previously hidden features enables routine change if these features provide affordances that are enacted in one or more organizational routines.

Inversion is of particular relevance for organizations that develop third-party modules to extend existing platform ecosystems, particularly if that inversion relates to features that are part of the platform core and are made visible through moving up in the design hierarchy. This is however not the focus of this paper which looks at how changes in platform ecosystems are related to changes in organizational routines of organizations that participate in that platform ecosystem. 
Table 1 summarizes these key relationships between applying modular operators at the ecosystem level and at the level of organizational routines. Opportunities for change originating in the platform ecosystem lead to augmentation or substitution of modules used within the organizational system as the organization seeks to capitalize on new or improved affordances.

Table 1. Enabling Ecosystem Impact

\begin{tabular}{|c|c|}
\hline Ecosystem Change & Routine Mirroring \\
\hline Augmentation & $\begin{array}{l}\text { Augmentation: } \\
\text { Enacting new affordance }\end{array}$ \\
\hline Splitting & $\begin{array}{l}\text { Augmentation: } \\
\text { Enacting new affordance }\end{array}$ \\
\hline Substitution & $\begin{array}{l}\text { Augmentation: } \\
\text { Enacting new affordance } \\
\text { Substitution: } \\
\text { Enacting improved affordance }\end{array}$ \\
\hline Inversion & $\begin{array}{l}\text { Augmentation: } \\
\text { Enacting new affordance }\end{array}$ \\
\hline
\end{tabular}

We did not attend to the operator of porting as it relates to "hidden modules"-porting describes the process where features are moved up the design hierarchy to be used within different modules [8]. There may thus be an indirect relationship as improved modules incorporating the ported module are made available (i.e., allowing for augmentation or substitution).

\subsection{Constraining Ecosystem Impact}

The first set of propositions is related to cases where changes in the ecosystem lead to the identification and enactment of novel affordances, and we have provided some examples from a fictious IIoT implementation. However, there are also situations where changes are made to the ecosystem that cast constraints on organizational routines.

First, if modules are removed from the ecosystem, it is likely that this exclusion will be mirrored in organizational routines as the discontinued availability of features and associated affordances can affect these routines. The discontinuance may limit the actions that have been part of past routine performances. The organization has to change their routines in order to ensure that they still meet their purpose. They might even decide to abandon affected routines altogether. Correspondingly:

Proposition 2a: The exclusion of ecosystem modules (removing modules from the system) can lead to constraints for organizational routines if the excluded module was used within those routines.
For instance, consider a certain module such as our predictive maintenance example to be discontinued and thus not available to a specific monitoring routine any longer. There are different ways for the organization to respond. First, they could turn to alternative modules, potentially from a different platform ecosystem, and substitute for the discontinued availability of technology-based affordances provided by the excluded module. Second, they might reorganize the routine to establish alternative ways of accomplishing the routine's goals - in the case of predictive maintenance this could mean that the organization goes back to using Excel or perhaps program their own solution in Python. Third, the routine could remain the same, but the discontinued affordance could lead to lower performance levels - in the predictive maintenance example they may simply stop doing predictive maintenance, in turn risking increased downtimes.

Constraints can also occur if a module is substituted and if the substitute provides a changed set of features:

Proposition 2b: Substituting ecosystem modules creates constraints if the substitution leads to a discontinuance of affordances previously enacted in one or more organizational routines.

For instance, a substituted predictive maintenance module may discontinue previously available affordances, thus requiring a change in a monitoring routine.

Table 2 summarizes these relationships.

Table 2. Constraining Ecosystem Impact

\begin{tabular}{|l|l|}
\hline Ecosystem Change & Routine Mirroring \\
\hline Exclusion & $\begin{array}{l}\text { Exclusion (from use within routine): } \\
\text { Adjustment of routine }\end{array}$ \\
\hline Substitution & $\begin{array}{l}\text { Substitution: } \\
\text { Adjustment of routine }\end{array}$ \\
\hline
\end{tabular}

Our model highlights how the modular operators are a useful device to describe how modular changes in platform ecosystems lead to changes within organizational routines. These changes can involve affordances and constraints for organizational routines. While new affordances provide opportunities for change, discontinued affordances or changed affordances involve constraints.

\section{Discussion \& Implications}

Changes in organizational routines can mirror developments in the platform ecosystem - be it voluntarily, for instance, as the organization makes sense 
of new modules and identifies relevant affordances, or because they are forced into that change, because it affects modules that are already in use.

This observation renders the organizational decision to participate in a specific ecosystem as a decision of strategic importance and potential risk. In an extreme case, the platform core may be discontinued, putting the entire networked system at risk-imagine a manufacturing organization that opted for one particular cloud solution to connect all there manufacturing equipment and open it up for additional services such as predictive analytics.

Over time, an organization using modules that are part of a platform ecosystem might experience opportunities for change as well as constraints which may lead them to change their organizational routines. This perspective involves a number of implications that may be related to (1) routine evolution, (2) the emergent properties of ecosystem-embedded organizational change, (3) decentralization of innovation, and (4) organizational as well as ecosystem pluralism. We discuss these in what follows.

\subsection{Platform Ecosystems \& Routine Change}

The evolution of organizational routines that capitalize on (i.e., enact) affordances provided by a platform ecosystem can be described in terms of coevolution of routines and ecosystems. This perspective casts a new light on organizational change, which becomes much less of an organizational level phenomenon, and increasingly dependent on exogenous influence from the organization's environment, not only in terms of its institutional environment but also the platform ecosystems the organization participates in. The key is that the organizational system partially mirrors the various ecosystems it participates in.

By attending to changes in platform ecosystems, it becomes clear that understanding routine change requires understanding the evolution of the platform ecosystem and how platform changes and routine changes interact. This assertion is consistent with previous research that has observed how changes in an ERP system lead to changes in routines [14]. While it has been argued that ERP systems can change routines in terms of substituting or altering actions, our model offers a more nuanced view on how changes in modern ecosystems can affect organizational work. First, changes in platform ecosystems, such as substitution of one module by a more advanced module (i.e., upgrade), may allow the organization to change activities in a routine, for instance, in order to improve on time or cost. Second, changes in platform ecosystems such as through augmentation or exclusion can entail disruptive change or, in some cases, even discontinuation of a routine or set of routines. Augmentation might provide features that create entirely new affordances that cause the organization to fundamentally rethink a routine or even abandon a routine in order to then introduce a new routine. Exclusion, on the other hand, may force the organization to fundamentally rethink a routine [27] or abandon a routine [28], because the routine is not feasible without the module formerly provided by the ecosystem. Finally, whereas existing studies tend to focus on situations where changes in routines are intended and mandatory $[9,13,14,29]$, we argue that routine change can evolve voluntarily when affordances are identified that are associated with new opportunities for organizational work.

Notably, the more frequent availability of new affordances (compared to using traditional software packages) might be one of the defining features of ecosystem-embedded change. Ecosystems are built on the idea that third party developers contribute modules and add affordances and the market creates incentives for the rapid development of new modules. Changes in ecosystems are more rapid than changes in traditional modular designs, such as the computer. Baldwin and Clark [8], for instance, wrote in 2003 when talking about the computer: "As one might expect, totally new augmentations are quite rare" (p. 137). This is not the case any longer, at least not with regards to platform ecosystems.

In our model, we attend to changes that originate in ecosystems, but one can also think of situations where routine change impacts on the ecosysteme.g., in cases where the organization in focus provides modules for the ecosystem or where the provider of modules and the organization in focus embark on co-innovation processes, or where simply customer feedback is incorporated in new versions of modules.

\subsection{Emergent Properties of Modular Chang- es and Mirroring in Routines}

Modular changes in ecosystems, and how they are mirrored in enactments in organizational routines, have yet another interesting implication for exploring routine change. Studies on how artifacts change the enactment of routines are commonly concerned with singular interventions, that is, a (digital) artifact is implemented at some point and organizational actors need to come to terms with it $[1,3,14,15,29]$. It has been found that change processes in routines unfold slowly as they involve, for example, recognizing change needs or opportunities, negotiating with other actors, and implementing changes [11]. The emphasis 
in existing studies, however, is on the implementation of artifacts - once they are in place, they are assumed to remain stable over time.

This is different for ecosystems. Once in place, ecosystems will be continuously configured and reconfigured. Ecosystems are not static. According to D'Adderio [1], a focus on how configurations of artifacts and actors challenge and establish patterns of interactions provides "valuable insights into routines micro-dynamics" (p. 211). In the following, we argue that studying modular change and routine mirroring can extend our understandings of routine change in three important ways.

First, since ecosystems change might occur rapidly while routine change takes time, there are potentials for conflicting overlaps. For example, ecosystems change might introduce new constraints or affordances (e.g., because bad customer feedback enforces rapid changes in the ecosystem) while actors are still engaging with sensemaking of previous changes (or they are implementing new practices based on identified affordances). In such cases, two practices may co-exist, or a newly established practice can be made obsolete.

Second, while we have assumed that actors intentionally change organizational routines in light of a persistent organizational goal, it could also be the case that new affordances of ecosystems lead to the emergence of new organizational goals. When actors collaborate in new ways (e.g. through the introduction of new modules in the form of boundary objects), they might develop new ideas and goals whilst interacting [30]. This, in turn, can also happen without prior intentions.

Finally, many organizations may draw on the same ecosystem(s). Thus, changes in the ecosystems manifest in different routines within different contexts. This could imply that ecosystems change across different organizations causes their practices and routines to converge over time. This might lead to "ecosystem-driven" isomorphism. At the same time, studying ecosystems change could also offer an opportunity to investigate how the same changes in one ecosystem lead to different change processes in different organizations. This, in turn, can extend our understanding of how micro-dynamics lead to routine change [1].

\subsection{Platform Ecosystems \& the Decentraliza- tion of Innovation}

Clearly, participating in a platform ecosystem and hence interweaving routines with platform ecosystem modules and their features and associated affordances decentralizes organizational change and innovation.
Not only routines are interwoven, but so are change activities as platforms and organizational routines coevolve. Organizational routines become dependent on the availability of and changes to ecosystem modules. The locus of value creation moves from inside the organization to outside [31].

From a design perspective, design decisions that impact on organizational routines are located both inside and outside the organization. While this phenomenon is not entirely new-organizations have always been using software systems that were subjected to change that can be described in terms of the modular operators - contemporary platforms provide the ground for the fluid evolution of platform ecosystems that lead to more distributed and more rapid innovation and change as ecosystems grow and their users capitalize on external network effects. This is particularly fostered by the deployment of modules in cloud-based systems, where modules can be frequently updated by providers. In a way, platform ecosystems are the full-fledged implementation of what Baldwin and Clark wrote about modular systems in general and the computer in particular: "... it is the nature of modular designs to tolerate the new and unexpected as long as the novelty is contained within the confines of a hidden module. Thus modular augmentations have been a persistent theme in the history of computers" (p. 137).

Attending to the role of change and innovation at the level of platform ecosystems is in response to recent calls for considering the external competitive environment in studies of digital innovation [32].

\subsection{Platform Ecosystems \& Pluralism}

There are at least two types of pluralism associated with platform participation that deserve our attention.

First, contemporary organizations are institutionally plural - that is, actors within organizations draw on multiple, nested, and sometimes contradictory institutional logics. This institutional context influence what affordances actors and groups of actors identify and how these affordances are enacted [26, 33]. Thus, the same ecosystem module and its features may be interpreted quite differently across the organization and thus play quite different roles across and even within organizational routines, if those routines span multiple fields of action.

Second, contemporary organizations typically participate in multiple platform ecosystems-just think of an enterprise that uses Microsoft products, has implemented an IoT solution for their production using Amazon's AWS, and also uses SAP. As a consequence, the organization is confronted with plural- 
istic, often competing opportunities to include and exclude modules from those competing platform ecosystems.

Together, these aspects suggest that a nuanced perspective is required to understand how ecosystem change and organizational change are interwoventhe organizational researcher must attend to both the pluralistic nature of organizational action and understand how these actions involve artifacts that originate from quite different environments, each with their own set of logics. Likewise, it suggests that practitioners need to simultaneously scan and observe changes in multiple environments which together provide technical artifacts use in organizational practice, and the various uses of those technologies in the pluralistic organizational context that shapes their daily routines and practices.

\section{Conclusion}

In this paper, we developed a conceptual framework suggesting that change in organizational routines in contemporary organizations is enmeshed with changes in the platform ecosystems the organization takes part in. On this view, organizational routines partially mirror the modular structures provided by the larger ecosystem and we see a new form of "embedded" organizational change that we call "routine mirroring." Changes in the ecosystem-in terms of core functionality, modules, interfaces, or regulations - translate into organizational changes as routine performances change.

One challenge in studying these changes is that contemporary organizations participate in, and are part of, multiple platform ecosystems at multiple levels including operating systems, enterprise systems, web-based systems, and others. This adds a new dimension to organizational pluralism. Not only draw contemporary organizations on multiple, sometimes conflicting institutional logics. Their routines heavily use information technologies that are embedded in broader platform ecosystems.

We suggest that contemporary theorizing on change and innovation involving digital technologies need to transcend organizational boundaries, and need consider processes of change and innovation that occur at the interface between organizations and the broader ecosystem they are part of, thus calling for multi-level theorizing encompassing both exogenous and endogenous triggers and processes of change and innovation.

\section{References}

[1] L. D'Adderio, "Artifacts at the centre of routines: Performing the material turn in routines theory," Journal of Institutional Economics, vol. 7, no. 2, pp. 197-230, 2011.

[2] M. S. Feldman and B. T. Pentland, "Reconceptualizing organizational routines as a source of flexibility and change," Administrative Science Quarterly, vol. 48, no. 1, pp. 94-118, 2003.

[3] B. T. Pentland and M. S. Feldman, "Narrative networks: Patterns of technology and organization," Organization science, vol. 18, no. 5, pp. 781-795, 2007.

[4] M. Ceccagnoli, C. Forman, P. Huang, and D. J. Wu, "Cocreation of Value in a Platform Ecosystem: The Case of Enterprise Software," MIS Quarterly, Article vol. 36, no. 1, pp. 263-290, 2012.

[5] A. Gawer, Platforms, markets and innovation. Edward Elgar Publishing, 2011.

[6] M. E. Porter and J. E. Heppelmann, "How smart, connected products are transforming competition," Harvard Business Review, vol. 92, no. 11, pp. 64-88, 2014.

[7] P. M. Leonardi, "When flexible routines meet flexible technologies: Affordance, constraint, and the imbrication of human and material agencies," MIS Quarterly, vol. 35, no. 1, pp. 147-168, 2011.

[8] C. Y. Baldwin and K. B. Clark, Design rules: The power of modularity. MIT press, 2000.

[9] B. T. Pentland and M. S. Feldman, "Designing routines: On the folly of designing artifacts, while hoping for patterns of action," Information and Organization, vol. 18, no. 4, pp. 235-250, 2008.

[10] J. Howard-Grenville and C. Rerup, "A process perspective on organizational routines," The SAGE handbook of organization process studies, pp. 323-337, 2016.

[11] K. Dittrich, S. Guérard, and D. Seidl, "Talking about routines: The role of reflective talk in routine change," Organization Science, vol. 27, no. 3, pp. 678-697, 2016.

[12] J. A. Howard-Grenville, "The persistence of flexible organizational routines: The role of agency and organizational context," Organization science, vol. 16, no. 6, pp. 618-636, 2005.

[13] L. D'Adderio, "The performativity of routines: Theorising the influence of artefacts and distributed agencies on routines dynamics," Research Policy, vol. 37, no. 5, pp. 769-789, 2008.

[14] O. Volkoff, D. M. Strong, and M. B. Elmes, "Technological embeddedness and organizational change," Organization Science, vol. 18, no. 5, pp. 832-848, 2007.

[15] P. Spee, P. Jarzabkowski, and M. Smets, "The influence of routine interdependence and skillful accomplishment on the coordination of standardizing and customizing," Organization Science, vol. 27, no. 3, pp. 759$781,2016$.

[16] V. L. Glaser, "Design performances: How organizations inscribe artifacts to change routines," Academy of Management Journal, vol. 60, no. 6, pp. 21262154, 2017.

[17] N. Berente, K. Lyytinen, Y. Yoo, and J. L. King, "Routines as shock absorbers during organizational transformation: Integration, control, and NASA's enterprise 
information system," Organization Science, vol. 27, no. 3, pp. 551-572, 2016.

[18] E. Cacciatori, "Resolving conflict in problem-solving: Systems of artefacts in the development of new routines," Journal of Management Studies, vol. 49, no. 8, pp. 15591585, 2012.

[19] H. Bapuji, M. Hora, A. Saeed, and S. Turner, "How Understanding-Based Redesign Influences the Pattern of Actions and Effectiveness of Routines," Journal of Management, p. 0149206317744251, 2018.

[20] S. Winter, N. Berente, J. Howison, and B. Butler, "Beyond the organizational 'container': Conceptualizing 21 st century sociotechnical work," Information and Organization, vol. 24, no. 4, pp. 250-269, 2014.

[21] A. Tiwana, B. Konsynski, and A. A. Bush, "Research Commentary--Platform Evolution: Coevolution of Platform Architecture, Governance, and Environmental Dynamics," Information Systems Research, vol. 21, no. 4, pp. 675-687, December 1, 20102010.

[22] K. Boudreau, "Does opening a platform stimulate innovation? The effect on systemic and modular innovations," SSRN eLibrary, 2007.

[23] A. Tiwana, "Evolutionary competition in platform ecosystems," Information Systems Research, vol. 26, no. 2, pp. 266-281, 2015.

[24] Y. Yoo, O. Henfridsson, and K. Lyytinen, "Research Commentary--The New Organizing Logic of Digital Innovation: An Agenda for Information Systems Research," Information Systems Research, vol. 21, no. 4, pp. 724-735, December 1, 20102010.

[25] M. L. Markus and M. S. Silver, "A foundation for the study of IT effects: A new look at DeSanctis and Poole's concepts of structural features and spirit," Journal of the Association for Information Systems, vol. 9, no. 10, pp. 609-632, 2008.

[26] S. Seidel and N. Berente, "Toward "third wave" information systems research: Linking sociomaterial practice with broader institutional logics," in 34th International Conference on Information Systems (ICIS 2013), Milan, Italy, 2013.

[27] S. Bucher and A. Langley, "The interplay of reflective and experimental spaces in interrupting and reorienting routine dynamics," Organization Science, vol. 27, no. 3, pp. 594-613, 2016.

[28] M. Fiol and E. O’Connor, "Unlearning established organizational routines-part I," The Learning Organization, vol. 24, no. 1, pp. 13-29, 2017.

[29] F. Iannacci and K. S. Hatzaras, "Unpacking ostensive and performative aspects of organisational routines in the context of monitoring systems: A critical realist approach," Information and Organization, vol. 22, no. 1, pp. 1-22, 2012.

[30] K. Dittrich and D. Seidl, "Emerging intentionality in routine dynamics: A pragmatist view," Academy of Management Journal, vol. 61, no. 1, pp. 111-138, 2018.

[31] G. Parker, M. Van Alstyne, and X. Jiang, "Platform ecosystems: How developers invert the firm," in Boston University Questrom School of Business Research Paper, ed, 2016.
[32] R. Kohli and N. P. Melville, "Digital innovation: A review and synthesis," Information Systems Journal, vol. 29, no. 1, pp. 200-223, 2018.

[33] L. Hultin and M. Mähring, "Visualizing institutional logics in sociomaterial practices," Information and Organization, vol. 24, no. 3, pp. 129-155, 2014. 